\title{
Was it justified?
}

\author{
John Le Heron
}

Received: 28 February 2011/ Accepted: 28 February 2011/Published online: 18 March 2011

(C) Australasian College of Physical Scientists and Engineers in Medicine 2011

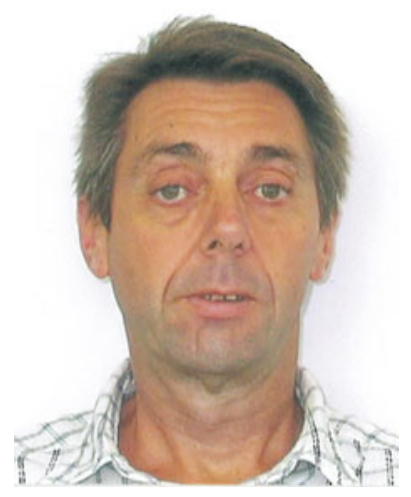

In many parts of the world, particularly in developing countries, the current International Basic Safety Standards for Protection against Ionizing Radiation and for the Safety of Radiation Sources (BSS) [1], published in 1996, has been the "bible" of radiation protection for the last decade or more, covering all aspects of radiation protection including medical exposure. A revision of the BSS commenced in 2007, culminating in a draft revised $\mathrm{BSS}^{1}$ that was given the green light at the end of 2010 to be allowed to progress through the final editorial stages leading to its publication. In this editorial I would like to reflect upon a particular issue pertinent to medical exposure that was grappled with during the drafting, namely

J. Le Heron ( $\square)$

Radiation Safety \& Monitoring Section, Division of Radiation, Transport and Waste Safety, International Atomic Energy

Agency, Wagramer Strasse 5, P.O. Box 100,

1400 Vienna, Austria

e-mail: J.Le.Heron@iaea.org justification of a particular radiological procedure for a given patient, primarily in the context of medical imaging.

The background to the revision of the BSS requirements for medical exposure is that the use of radiation in medicine has continued to grow in the 15 years since the publication of the BSS. It is not just a simple case of more equipment being available for use throughout the world. There are also changes in techniques and technologies available to be used-digital imaging, multi-detector CT, PET-CT hybrid imaging, and image guided interventional procedures, for example. In the case of imaging there are also changes in the roles that medical exposures play-the imaging department is often a first port of call for diagnosis, and screening in all its guises is playing a bigger role in the practice of medicine.

The requirements in the BSS and the revised BSS are based on the recommendations of the International Commission on Radiological Protection (ICRP), and clearly embrace the 3 ICRP principles of radiation protectionjustification of the practice, optimization of the protection and dose limitation. Only the first two apply to medical exposure-dose limitation does not apply to medical exposure. But of these two radiation protection principles, there appears to be an imbalance in how well each is being implemented in daily practice.

The concept of justification as it applies to medical uses of radiation is reasonably well established, with the current 3-level approach having been introduced with the ICRP Publication 73 [2] and more recently reiterated in ICRP Publications 103 [3] and 105 [4]. However the transfer into day-to-day practice has proven more difficult, especially

\footnotetext{
${ }^{1}$ Current draft of the BSS is at: http://www-ns.iaea.org/committees/ bss/default.asp?fd=1035
} 
with respect to "level 3" justification for an individual patient undergoing a given radiological procedure. In contrast to the principle of optimization of radiation protection where there are many well established tools for its implementation, the implementation of justification has remained very much in its infancy. The evidence for the ineffective application of the principle has been gaining greater publicity in recent years, with many media reports appearing on inappropriate, unnecessary or unjustified radiological procedures. Why is there this problem with justification of radiological procedures, especially with imaging procedures?

To discuss the problem it is first necessary to consider two key roles that are involved with patients undergoing radiological procedures. The first is that of the "referrer"the doctor, whose care the patient is under, who refers the patient for a given radiological procedure. The second role is that of the doctor under whose responsibility the actual radiological procedure will take place. The revised BSS has introduced two terms to cover these roles-the referring medical practitioner and the radiological medical practitioner. In the context of imaging, the referring medical practitioner is typically, but not limited to, the general practitioner or specialist, while the radiological medical practitioner is usually, but again not limited to, the radiologist. In some situations both roles are fulfilled by the same person and, in some circumstances, this self-referral has been identified as one of the reasons for unnecessary or unjustified radiological procedures.

Which of these two roles is more appropriate for performing the justification of a given radiological procedure for a given patient? As the draft revised BSS evolved, formal comments were received at various stages of its development. The requirements on justification in medical exposure elicited a large number of comments, especially with respect to this question. In essence the comments ranged from supporting the draft text which had the primary responsibility with the radiological medical practitioner and with the referring medical practitioner in support, through to comments that stated it should be the other way around. When analysed more closely, the comments could be synthesised as follows.

The referring medical practitioner has the specialist knowledge about the patient and their medical history and hence the context for the proposed radiological procedure, but typically has little or even no knowledge about the radiation doses, risks and limitations of the procedure. On the other hand, the radiological medical practitioner has the specialist knowledge about the radiological procedure, including its benefits, risks and limitations, but is lacking the in-depth knowledge about the patient. Thus, at face value each role would have a legitimate claim for responsibility, though neither has the full story.
But there are more complications. Depending on the health system of a given country, the radiological medical practitioner may have a financial interest in performing the radiological procedure-the more procedures, the more income. This would suggest that the radiological medical practitioner should not be the gatekeeper. However the independence of the referring medical practitioner may also be questioned, especially if they are working in a country where there is a culture of medical litigation leading to the practice of defensive medicine-a case of requesting more radiological procedures than strictly necessary, in the hope of covering all contingencies. The presence of a litigious culture in a country may also cause a given professional group, such as radiologists, to avoid taking responsibility for justification, simply as a means of minimizing the risk of being sued i.e. they would prefer to "pass the buck".

There are societal and cultural differences to the practice of medicine and in particular to the use of radiation for imaging. In some countries, the request for a radiological procedure from a referring medical practitioner is viewed as a request for a consultation with a specialist, namely the radiological medical practitioner. However in other countries, the request for a radiological procedure is viewed as an order or instruction from the referring medical practitioner to the radiological medical practitioner to perform the procedure. Clearly these relationships impact on how and by whom the justification is to be performed.

In several countries professional bodies have produced guidance on imaging procedures, including when they should be performed, when they should not be performed, advantages, limitations and radiation risk. These so-called referral guidelines or appropriateness criteria are a useful bridge between the radiological medical practitioner and the referring medical practitioner.

The final decision for the revised BSS was that "level 3" justification should be a joint responsibility, performed by consultation between the radiological medical practitioner and the referring medical practitioner. Clearly the use of accepted referral or appropriateness criteria will be a crucial tool in this process, and the incorporation of such criteria into future electronic referral systems augurs well for a better implementation of justification in practice.

How else can the implementation of level 3 justification be improved? Approaches that are being explored by the International Atomic Energy Agency include promoting the "3 A's" - namely, awareness, appropriateness and audit. The level of awareness of radiation risk among health professionals is so low that it is insufficient to allow effective justification to take place. Awareness clearly needs to be improved. As mentioned above, referral or appropriateness criteria are available, but their dissemination and use needs to be improved throughout the world. 
Audit should be an effective tool for ensuring that justification is practised, and the promotion of its use could also favourably impact on minimizing numbers of inappropriate examinations.

Another recent development is the promotion of the idea of tracking patient doses. This is in response to the increasing number of diagnostic and image guided interventional procedures that an individual patient may undergo in a few years or during a life time, with the implication that knowledge of cumulative patient doses will improve the implementation of justification. Easy availability of patient medical history, including radiological procedures, should contribute to better implementation of justification, including a reduction in procedures unnecessarily repeated when moving from one facility to another. But how knowledge of cumulative patient dose will help is less clear. Is there a value of cumulative dose beyond which the risks have become unacceptable and further exposure would be unjustified? This is certainly the case for occupational exposure, where dose limits (and implicitly cumulative dose) are the final means for ensuring a level of protection deemed acceptable. However there are no dose limits for medical exposure. Perhaps the magnitude of the cumulative patient dose could determine the degree of rigour in the justification process. As noted already in the editorial, the level of implementation of justification is low, even in its most rudimentary form. A cumulative dose-based system may be a step too far at the moment, but perhaps is a possibility for the future.
To summarize, the revised BSS establishes a clearer framework for the implementation of the justification principle for medical exposures and it is hoped that through the initiatives such as the 3 A's there will be a positive impact on day-to-day practice.

The views expressed in this editorial are those of the author and should not be interpreted as describing the position of the IAEA.

\section{References}

1. Food and Agricultural Organization of the United Nations, International Atomic Energy Agency, International Labour Organization, OECD Nuclear Energy Agency, Pan American Health Organization, World Health Organization, International Basic Safety Standards for Protection against Ionizing Radiation and for the Safety of Radiation Sources (1996) Safety Series No. 115, IAEA, Vienna

2. International Commission on Radiological Protection, Radiological Protection and Safety in Medicine (1996) ICRP Publication 73, Ann. ICRP 26(2). Pergamon Press, Oxford

3. International Commission on Radiological Protection, The 2007 Recommendations of the International Commission on Radiological Protection (2007) ICRP Publication 103, Ann. ICRP 37(2-4). Pergamon Press, Oxford

4. International Commission on Radiological Protection, Radiological Protection in Medicine (2007) ICRP Publication 105, Ann. ICRP 37(6). Pergamon Press, Oxford 